\title{
Salty \& Sweet: Are Salt and Free Sugars Added to Commercial Complementary Baby Foods Currently Available for Purchase in New Zealand? ${ }^{+}$
}

\author{
Shanjivan Padarath ${ }^{1}$, Sally Mackay ${ }^{2, *}$ and Sarah Gerritsen ${ }^{2}$ \\ 1 School of Population Health, University of Auckland, Auckland 1072, New Zealand; \\ spad267@aucklanduni.ac.nz \\ 2 Department of Epidemiology \& Biostatistics, School of Population Health, University of Auckland, \\ Auckland 1072, New Zealand; s.gerritsen@auckland.ac.nz \\ * Correspondence: sally.mackay@auckland.ac.nz; Tel.: +64-9-923-8733 \\ † Presented at the 2019 Annual Meeting of the Nutrition Society of New Zealand, Napier, New Zealand, 28- \\ 29 November 2019.
}

Published: 13 December 2019

The New Zealand Ministry of Health infant and toddler guidelines (2008) recommend careproviders "choose pre-prepared complementary foods with no added salt, fat, honey or other sweeteners" to promote healthy taste preference development and protect child physical and oral health. This study aimed to determine whether commercial complementary baby foods (CCBFs) available for purchase in New Zealand supermarkets contained added salt and free sugars.

Data are from Nutritrack - a database of all packaged food/beverages sold at the four major supermarkets in New Zealand in 2019. Products were classed as either breakfasts, meals, desserts, or snacks based on product name and ingredient list. Ingredient lists were analyzed to identify the presence of added salt and free sugars (monosaccharides, disaccharides, fruit juices and fruit juice concentrates). Sodium content was reviewed against the Codex Alimentarius guidelines to identify baby foods with excess sodium.

Of the 198 CCBFs, eight products (4\%) contained added salt. Half of these $(n=4)$ were for infants, 3 for toddlers and 1 product had no specific age. Five were snacks, two were dry cereals, and one was a vegetable-based meal. Two of these CCBFs along with eight others (5\%) had a high sodium content. Furthermore, sixty-eight products (34.3\%) contained free sugars. Fifty were for infants, 17 for toddlers and 1 had no specified age. All the desserts $(n=21), 57.1 \%$ of snacks $(n=20), 31.3 \%$ of breakfasts $(n=$ $10)$, and $15.5 \%$ of meals $(n=17)$ contained free sugars.

Only a small portion of CCBFs contained added salt and were high in sodium content. However, more than a third of CCBFs contained added free sugars; which may be detrimental to infants and toddlers body size, development of taste preferences and oral health. These results can inform the current revision of the infant and toddler dietary guidelines and potentially, policy development regarding product formulation.

(C) 2019 by the authors. Licensee MDPI, Basel, Switzerland. This article is an open access article distributed under the terms and conditions of the Creative Commons Attribution (CC BY) license (http://creativecommons.org/licenses/by/4.0/). 